\title{
Collimation of laser-produced plasmas using axial magnetic field
}

\author{
AMITAVA ROY,${ }^{1,2}$ SIVANANDAN S. HARILAL, ${ }^{3}$ SYED M. HASSAN, ${ }^{1}$ AKIRA ENDO, ${ }^{2}$ \\ TOMAS MOCEK, ${ }^{2}$ AND AHMED HASSANEIN ${ }^{1}$ \\ ${ }^{1}$ School of Nuclear Engineering and Center for Materials Under Extreme Environment, Purdue University, West Lafayette, Indiana \\ ${ }^{2}$ HiLASE Centre, Institute of Physics ASCR, Dolní Břežany, Czech Republic \\ ${ }^{3}$ Pacific Northwest National Laboratory, Richland, Washington
}

(RECEIVED 2 September 2014; AcCEPTED 6 January 2015)

\begin{abstract}
We investigated the expansion dynamics of laser-produced plasmas expanding into an axial magnetic field. Plasmas were generated by focusing $1.064 \mu \mathrm{m} \mathrm{Nd}$ :YAG laser pulses onto a planar tin target in vacuum and allowed to expand into a $0.5 \mathrm{~T}$ magnetic field where the field lines were aligned along the plume expansion direction. Gated images employing an intensified charge-coupled device showed focusing of the plasma plume, which were also compared with results, obtained using particle-in-cell modeling methods. The estimated density and temperature of the plasma plumes employing emission spectroscopy revealed significant changes in the presence and absence of the $0.5 \mathrm{~T}$ magnetic field. In the presence of the field, the electron temperature is increased with distance from the target, while the density showed opposite effects.
\end{abstract}

Keywords: Laser-produced plasma; Optical emission spectroscopy; Plasma-B field interaction; Plasma temperature and density; Tin plasma

\section{INTRODUCTION}

The interaction of highly transient laser-generated plasmas with magnetic $(B)$ fields is a fascinating area as the $B$-field can be used to manipulate the dynamic properties of these expanding plasmas (Schwarz \& Hora, 1971; Dimonte \& Wiley, 1991; VanZeeland et al., 2001; Harilal et al., 2004). The plasma- $B$ field interaction studies are of relevance to astrophysical jets, the propagation of charged particle beams, solar wind evolution, bipolar flows associated with young stellar objects, laser-induced breakdown spectroscopy, pulsed laser deposition (PLD), etc. (VanZeeland et al., 2001; Shen et al., 2006; Ye et al., 2013). In the field of inertial fusion, confinement of the expanding target plasma using a $B$-field offers a potential means to slow high-energy particles before they implant in the surrounding structures. The $B$-field is also used for controlling or diverting ion debris in extreme ultraviolet (EUV) lithography laser-produced plasma (LPP) sources (Harilal et al., 2006; Roy et al., 2014a; 2014b).

Address correspondence and reprint requests to: Amitava Roy, School of Nuclear Engineering and Center for Materials Under Extreme Environment, Purdue University, West Lafayette, Indiana 47907, USA. E-mail: roy @ fzu. cz, aroy@ barc.gov.in and Sivanandan S. Harilal, Pacific Northwest National Laboratory, Richland, Washington 99352, USA. E-mail: hari@pnnl.gov
Also it has been reported that droplet-free thin films can be fabricated with the use of a $B$-field in PLD (Tsui et al., 2002).

The $B$-field presence during LPP expansion may initiate several interesting physical phenomena depending on the orientation of the field lines. Previous studies employing a LPP expanding into a transverse $B$ field showed that conversion of the plasma thermal energy into kinetic energy, plume confinement, ion acceleration, emission enhancement, plasma instabilities, etc. (Neogi \& Thareja, 1999; Rai et al., 2003; Harilal et al., 2004; 2005a; 2005b; Pagano \& Lunney, 2010; Kumar et al., 2011a; 2011b; 2012; Pandey \& Thareja, 2011). A $B$-field is capable of providing such a control by influencing the dynamics, ionization, and optical properties of a LPP. High $\beta$ ( $\beta=$ plasma pressure/magnetic pressure) LPPs surrounded in ambient magnetized plasmas provide a laboratory simulation of various astrophysical phenomena, viz; supernova explosions, coronal mass ejection, magnetic reconnection in solar flares, etc. (VanZeeland et al., 2003; Gekelman et al., 2007). Axial $B$ fields are also an efficient method for guiding the ion streams from laser plasmas.

The effect of varying the transverse $B$-field on the plasma plume emission of laser-produced lithium plasma has been investigated using an atomic analysis (Joshi et al., 2010). The effect of a transverse $B$-field on the laser-blow-off 
plasma plume emission also has been studied in the presence of an ambient gas (Kumar et al., 2011a; 2011b). Enhancement in the intensity associated with structures was observed in the temporal profile of neutrals, which was strongly dependent on the $B$-field intensity, distance from the target and background gas pressure.

Laser-produced tin plasma has come up as a most promising and energy-efficient next generation EUV light source for the semiconductor lithography industry (Bakshi, 2009; Roy et al., 2014a; 2014b). A high-brightness and debris-free source emitting at $13.5 \mathrm{~nm}$ radiation with $2 \%$ bandwidth (in-band) is necessary for this purpose. Hot tin plasmas produced by intense laser pulses heated to $\sim 30 \mathrm{eV}$ emit-efficient EUV radiation in the in-band region (Yuspeh et al., 2011). Apart from conversion efficiency of laser energy to EUV photons, the cleanness of the EUV LPP sources is also extremely important for their use in semiconductor lithography (Coons et al., 2010). The EUV LPP source emits debris in the form of energetic ions, atoms, and molten droplets $(\mathrm{Wu}$ et al., 2012). Several schemes are proposed for mitigating the ion and atom debris, which includes ambient gas, magnetic field, combination of both magnetic field and ambient gases, mass-limited targets, electrostatic repeller fields, etc. (Bakshi, 2009). By applying a static $B$-field of about $1 \mathrm{~T}$, a fivefold reduction in $\mathrm{Sn}$ debris deposited on small $\mathrm{Mo} / \mathrm{Si}$ multilayer mirrors from an $\mathrm{Sn}$ planar target plasma has been demonstrated (Ueno et al., 2008). The design of a mitigation system is simulated with the computer models with general heterogeneous target systems integrated package (Sizyuk et al., 2007). Apart from guiding ions, the magnetic field can also influence the plasma characteristic parameters (density and temperature).

In this paper, we report the dynamics of a LPP expanding into a $0.5 \mathrm{~T}$ axial magnetic field. Gated images employing intensified charge-coupled device (ICCD) showed collimation and focusing of the plasma plume which were compared with results obtained using particle-in-cell (PIC) modeling. We performed optical emission spectroscopy (OES) analysis of LPP in the presence and absence of $B$-field to characterize the plume parameters. Our results show that the plasma fundamental parameters are significantly altered in the presence of $0.5 \mathrm{~T} B$-field.

\section{EXPERIMENTAL SETUP}

Experiments were carried out using a $Q$-switched Nd:YAG laser emitting at $1.064 \mu \mathrm{m}$ in wavelength with a pulse duration $\sim 5 \mathrm{~ns}$ full width half maximum (FWHM). Details of the experimental setup can be found elsewhere (Roy et al., $2014 a ; 2014 b)$. The laser beam energy was attenuated using a combination of a half-wave plate and a polarizing cube. Planar slab of pure Sn was mounted to a servo-motor controlled $X Y Z$ translation stage inside a stainless steel highvacuum chamber. The target was placed parallel to the base plate of the chamber and the laser angle of incidence used was $66^{\circ}$ with respect to the target normal. The plasma plume expanded in a direction normal to the target (and parallel to magnetic field). The details of the $B$-field configuration and distribution obtained using a Gauss meter are given elsewhere (Roy et al., 2014a; 2014b). The target was placed in a chamber which was evacuated using a turbomolecular pump to a pressure lower than $10^{-5}$ Torr. A $40 \mathrm{~mm}$ planoconvex focusing lens was mounted on micrometer actuator controlled $X$-translation stage, which allowed tunability of the laser spot size. Laser focal spot employed in the experiment was $80 \mu \mathrm{m}$. The target was translated between shots to refresh the surface, and to mitigate the effects of target cratering. The beam energy was monitored using an energy meter.

The plume imaging was accomplished using an ICCD placed orthogonal to the plasma expansion direction. A Nikon objective lens was used to image the plume region onto the camera to form a two-dimensional (2D) image of the plume intensity. The visible radiation from the plasma was recorded integrally in the wavelength range $350-900 \mathrm{~nm}$. A programmable timing generator was used to control the delay time between the laser pulse and the imaging system with overall temporal resolution of $1 \mathrm{~ns}$. For performing space resolved OES, the self-emission from the plasma was collected and imaged onto the slit of a $0.5 \mathrm{~m}$ spectrograph (max resolution $0.03 \mathrm{~nm}$ ). The ICCD was used as a detector to collect the dispersed light. The slit of the spectrograph was aligned parallel to the magnetic field or to the plasma expansion direction. The spectrograph was operated in the imaging mode, which gives spectral information with high spatial precision.

\section{RESULTS AND DISCUSSION}

The presence of a $B$-field during the generation of laser-plasma will affect its fundamental parameters as well as its expansion hydrodynamics. We used fast photography employing an ICCD which provides 2D snapshots of the 3D plume propagation. These images provide valuable in sight into the hydrodynamic expansion of plasma plumes, as well as radiation emission. Typical gated images recorded in the presence and the absence of $B$-fields are given in Figure 1. The integration time used is $2000 \mathrm{~ns}$. One can see that the shape of the plume changes significantly in the presence of $0.5 \mathrm{~T} B$-field. In the absence of $B$-field, the plasma plume expands freely and adiabatically. In the presence of $B$-field, significant improvement in plume emission and guiding of the plasma along the field lines were observed. The colors in the ICCD camera images indicate different radiation intensity values where white represents maximum counts. Higher counts observed in the images correspond to areas of higher temperature and/or particle density in the plasma plume. Plasma plume becomes much brighter in the presence of the $B$-field indicating increase in plasma temperature. Secondary plasma formed on the opposing magnet is also visible in the image. This could be caused by fast electrons and ions emanating from the plasma hitting 


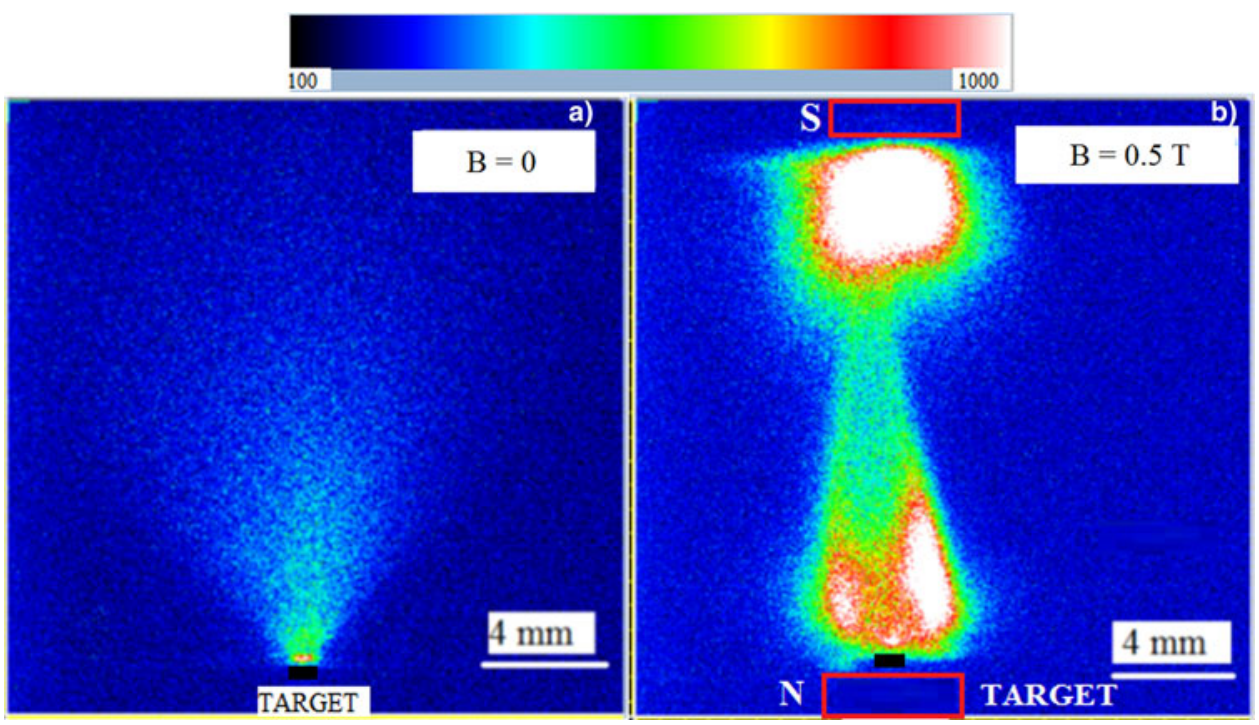

Fig. 1. Time integrated plume images are given (a) in the absence (b) and presence of axial $B$-field. The laser energy and spot size were $30 \mathrm{~mJ}$ and $80 \mu \mathrm{m}$ in diameter, respectively. The black rectangle represents position of the tin planar target and the red rectangle displays the position of the magnet poles.

the top magnet (Roy et al., 2014a; 2014b). The lateral peak plume expansion velocities measured from the images are 1.2 and $0.75 \mathrm{~cm} / \mu$ s without and with the axial $B$-field, respectively. Therefore, with the application of a $0.5 \mathrm{~T} B$-field the plasma plume expansion velocity reduces by a factor of 1.6 in the lateral direction.

The plume expansion features recorded using fast photography in the presence and absence of $0.5 \mathrm{~T}$ axial $B$-field are simulated using PIC code (Balk, 2007; Roy et al., $2014 a ; 2014 b$ ). Figure 2 displays the ion and electron motion and respective energies at real time $\sim 271 \mathrm{~ns}$ with $0.5 \mathrm{~T}$ axial static $B$-field. One can see from Figure 2 that the plasma particles are confined in the axial direction. Hence our simulation results qualitatively predict the plasma behavior observed in Figure 1. The self-emission image given in Figure 1 recorded in the visible spectral region (350-900 nm) in a timeintegrated manner with a gating time of $2000 \mathrm{~ns}$. However, it has to be mentioned that, the plasma plume also emits in the other spectral windows (e.g., UV) and hence Figure 1 is not the full representation of entire plume emission feature.

The lateral confinement of the plasma is clearly visible in the ICCD and PIC images. Therefore, one can expect plasma heating and changes in density due to collimation and focusing effect. Hence, we performed OES studies to estimate the fundamental plume parameters in the presence and absence of $B$-field. Such spectroscopic studies provide much insight into the context of plasma- $B$ field interaction.

We performed OES analysis of plasma in the presence and absence of $0.5 \mathrm{~T}$ axial $B$-field. For recording the dispersed light from the plasma, the slit of the spectrograph was aligned parallel to the $B$-field (plasma expansion direction) and the ICCD was operated in the imaging mode. Using $2 \mathrm{D}$ imaging, it was possible to obtain plasma emission spectra with high spatial precision $(25 \mu \mathrm{m})$. Typical 2D spectral images recorded from the tin plasma in the presence and absence of $B$-field is given in Figure 3. These images were obtained with $2000 \mathrm{~ns}$ integration time starting from the onset of the plasma generation. Figure 3 shows that the spatial extension of emission lines was altered in the presence of $0.5 \mathrm{~T}$ field. It is also interesting to note that the spatial extent of Sn II line emission at 556.19 and $558.89 \mathrm{~nm}$ are more or less similar in the presence of the $B$-field compared with field-free case. Some Sn III lines (534.9 and $536.94 \mathrm{~nm}$ ) are found to be extended to larger distances with the application of the axial $B$-field. One can also see a significant increase in continuum radiation in the presence of $B$-field. As seen in the ICCD imaging and PIC modeling results (Figs 1 and 2), the applied axial magnetic field confines the plasma greatly. Hence the enhanced emission in the presence of a magnetic field could be due to increase in the rate of various recombination mechanisms in the plasma (Rai et al., 2003).

The ICCD images given in Figure 1 show that the spatial extension and lifetime of the plasma are significantly increased with the application of $B$-field. However, the spectral image given in Figure 3 is not showing that significant difference. This could be due to the fact that the plume is confined laterally and wings are emitting more compared with the plume central position (orthogonal to the laser spot) where the spectral imaging study was performed. Moreover, the self-emission images given are spectrally integrated (350-900 nm) while 2D spectral images are wavelength resolved. Figure 4 displays a typical OES spectra measured at a distance of $1 \mathrm{~mm}$ from the tin target. One can see from Figure 4 that, with the application of a $B$-field the intensities of certain lines along with background continuum are enhanced, whereas some line intensities are suppressed. Prominent lines seen in the spectra include Sn II at $533.24,556.19,558.89 \mathrm{~nm}, \mathrm{Sn}$ I at $563.17 \mathrm{~nm}$, and Sn III at $534.9 \mathrm{~nm}$. It can be seen from the spectra that the intensity of 


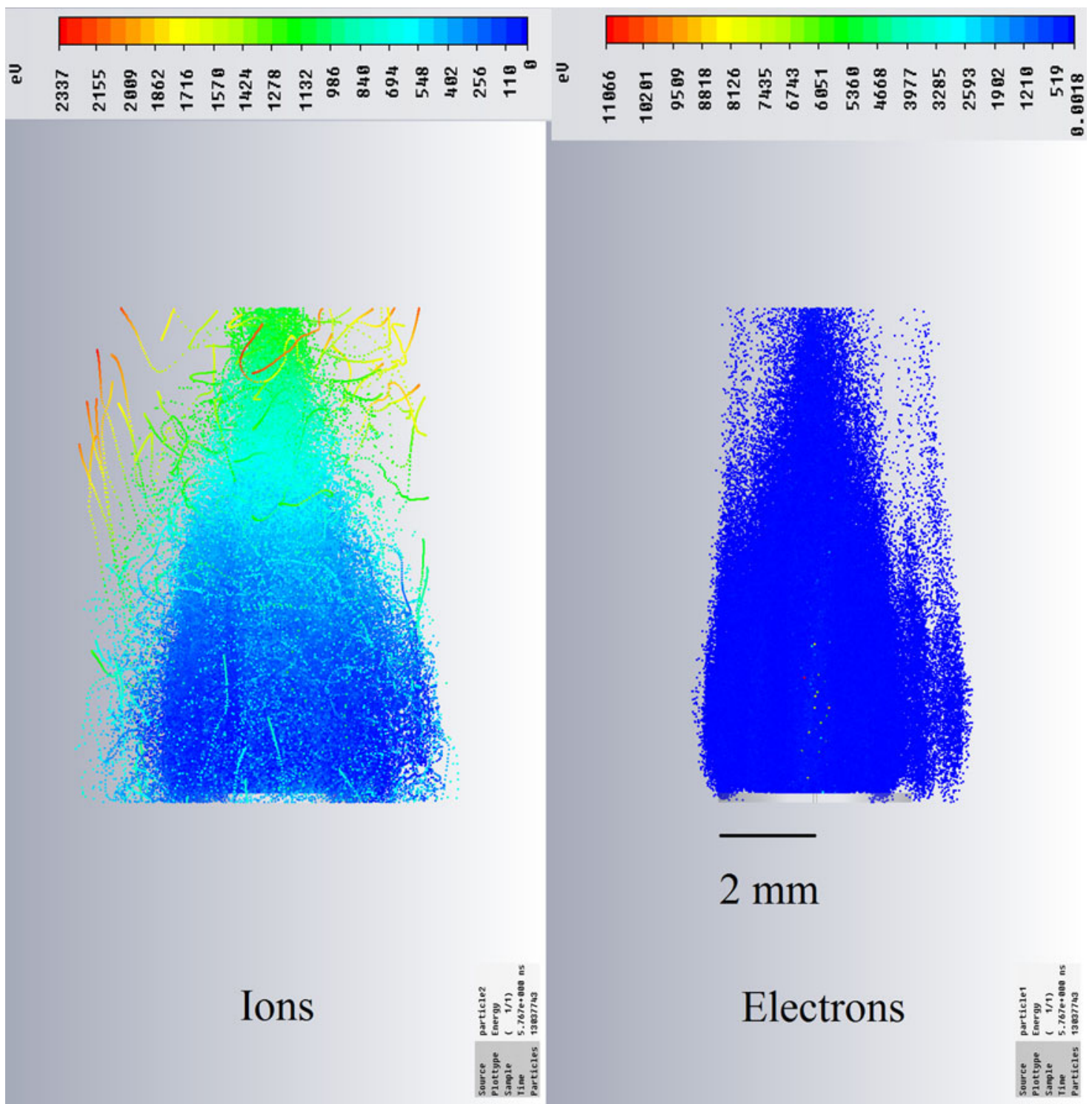

Fig. 2. A snap shot of ion and electron motion from PIC simulation at $\sim 271 \mathrm{~ns}$ in real time with $0.5 \mathrm{~T}$ axial magnetic field. The line colors in the picture represent energy of the particles.

the ionic lines increased significantly with $B$-field presence, while the changes are minimal for neutral lines. Interestingly, for transverse magnetic field case the ionic spectral line does not exhibit any significant change with both, magnetic field and ambient gas (Kumar et al., 2011a; 2011b).

Figures 5 and 6 show the line intensities of Sn I $(563.17 \mathrm{~nm})$ and $\mathrm{Sn}$ II $(556.19 \mathrm{~nm})$ with distance from the target. One can see from these figures that ionic line intensities are enhanced with the application of a $B$-field, whereas the neural lines have no significant intensity changes. Contribution from various atomic processes (electron impact excitation, field-induced ionization, etc.) needs to be considered carefully in order to find out the change in emission due to magnetic field (Joshi et al., 2010). The neutrals do not interact with the $B$-field as do the ions therefore it is expected that the neutral lines will not be affected by the application of an axial magnetic field. However, the $B$-field confinement of the plasma will affect the excited populations of all species in the plume. One can see from Figure 5 that there is an increase in neutral Sn line intensities with $B$-field at short distances. Assuming three-body recombination as the major mechanism, which has got $n_{\mathrm{e}}^{2}$ dependence, it will be prevalent at short distances. Figure 6 displays that the Sn II line intensities have maximum around $0.3 \mathrm{~mm}$ and then it decreases.

We also employed OES to obtain the space evolution of the fundamental plasma quantities of the tin plume; viz., electron temperature $\left(T_{\mathrm{e}}\right)$ and density $\left(n_{\mathrm{e}}\right)$. Excitation temperature was obtained by invoking local thermodynamic equilibrium (LTE) arguments and generating Boltzmann diagrams, while electron density was estimated from Stark broadening of isolated lines (Griem, 1997). In optically thin plasma, the relative intensity $I_{i j, k}$ of the lines emitted from a given state of 

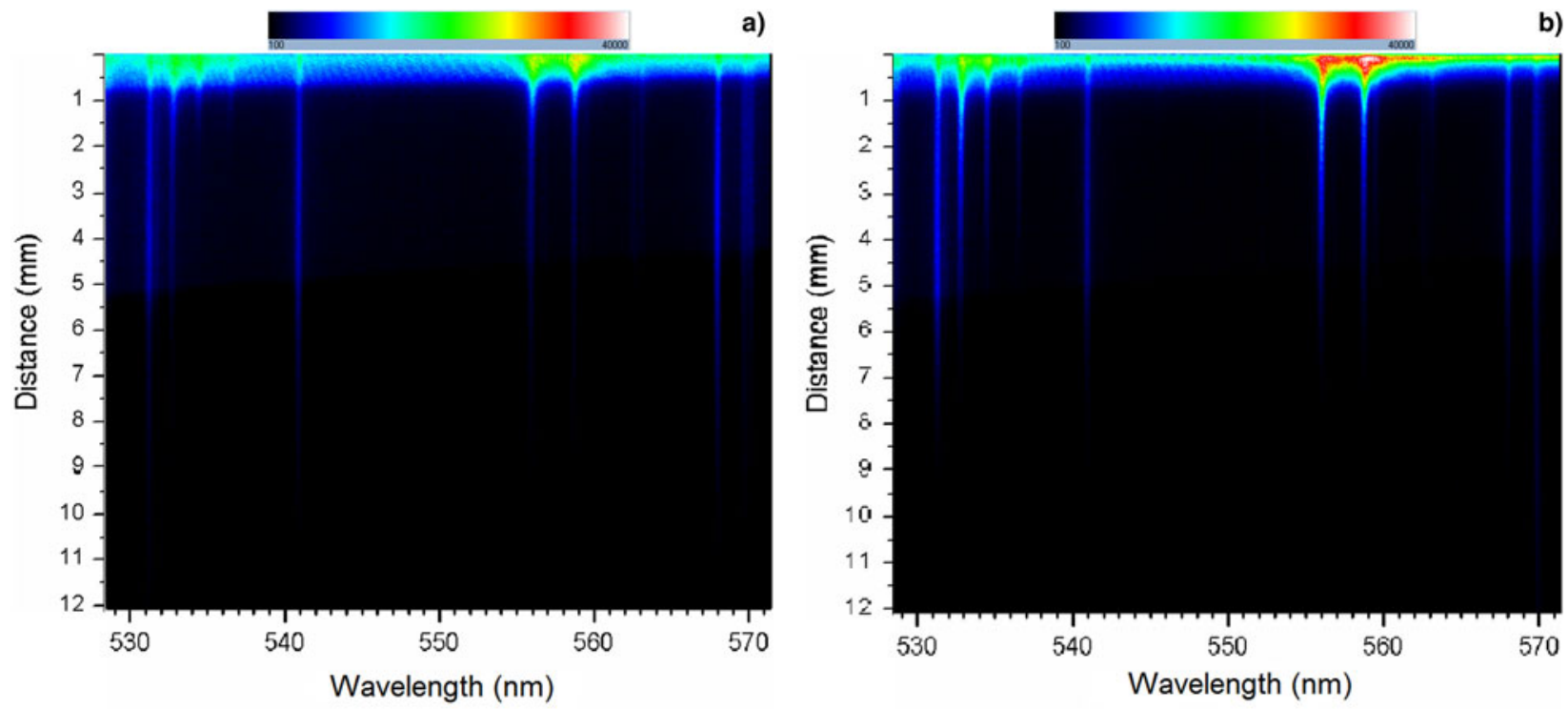

Fig. 3. $2 \mathrm{D}$ spectral images of tin plasmas for $30 \mathrm{~mJ}$ laser energy (a) in the absence and (b) in the presence of $0.5 \mathrm{~T}$ magnetic field. The images were taken at the onset of the plasma spark and were integrated from 0 to $2000 \mathrm{~ns}$. The laser intensity is $1.2 \times 10^{11} \mathrm{~W} / \mathrm{cm}^{2}$.

excitation can be used to calculate the excitation temperature, if the transition probabilities $A_{i j}$ and the statistical weight of the upper level $g_{i}$ are known, via the expression for a transition from a higher state $i$ to a lower state $j$. using the following relation:

$$
\ln \left(\frac{I_{i j, \lambda} \lambda_{i j}}{F_{\lambda} g_{i} A_{i j}}\right)=-\frac{1}{k_{\mathrm{B}} T} E_{i}+\ln \left(\frac{h c L n_{z}}{4 \pi P_{z}}\right)
$$

The subscript $z$ refers to the ionization state of the species $(z=0$ and 1 correspond to neutral and singly ionized atoms, respectively), $P_{z}$ is the partition function, $n_{z}$ is the number density of the emitting species, $L$ is the plasma

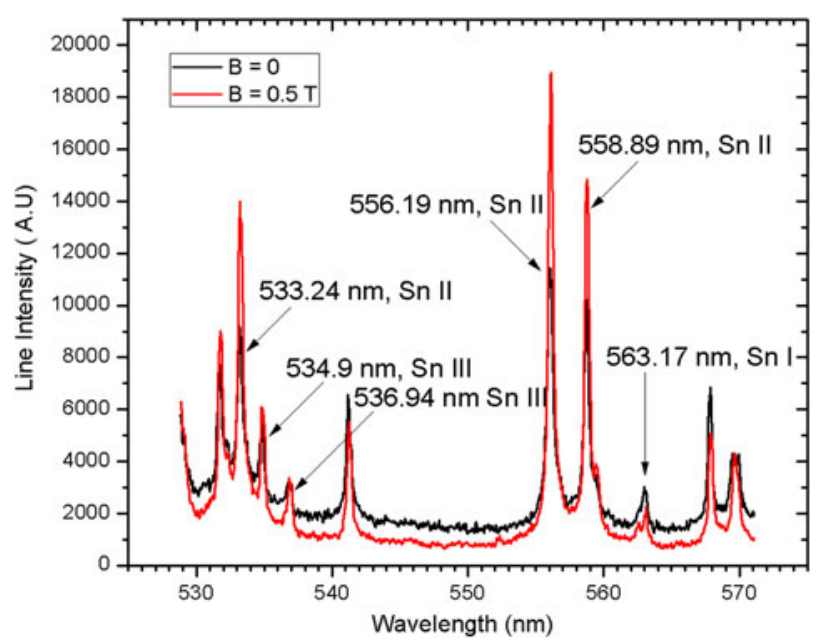

Fig. 4. The OES spectra of tin plasma at a distance of $1 \mathrm{~mm}$ from the target in the presence and absence of axial $B$-field. characteristic length. $F_{\lambda}$ is the calibration factor of the spectrograph. The plasma temperature was estimated from the Boltzmann plot of Sn II emitting at 533.24, 556.19, 558.89, and $563.17 \mathrm{~nm}$ for which absolute transition probabilities are well known (NIST data base).

Figure 7 displays the plasma temperature with respect to distance from the target in the presence and absence of $B$-field. In the field-free case near the target surface, the electron temperature $T_{\mathrm{e}}$ initially increased to $2.2 \mathrm{eV}$ when the axial distance was increased to $1 \mathrm{~mm}$ and then decreased slowly. The reduction in $T_{\mathrm{e}}$ with distance is related to free adiabatic expansion of the plasma in space.

One can see from Figure 7 that electron temperature increases by a factor of 1.5 in the presence of $0.5 \mathrm{~T}$ axial

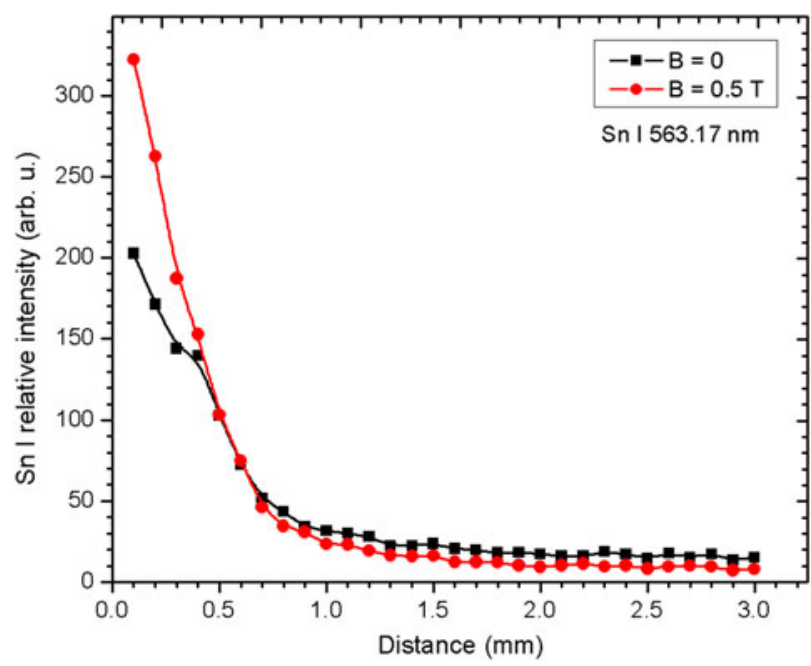

Fig. 5. The measured neutral Sn I line intensities with respect to distance from the target. 


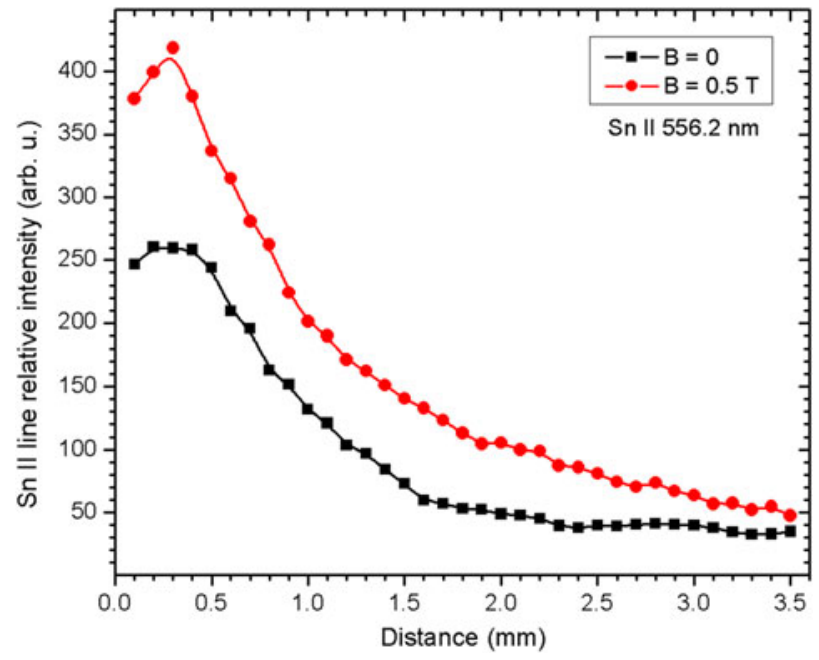

Fig. 6. The measured Sn II line intensities with respect to distance from the target.

$B$-field and the $B$-field has less influence at short distances. Similar increase in $T_{\mathrm{e}}$ has also been reported for carbon plasma when expanding across a transverse $B$-field (Neogi $\&$ Thareja, 1999). In case of the transverse $B$-field, surface currents are set up due to finite resistivity at the plasma field boundary, producing an increased temperature on the account of Ohmic heating. In the present experiment, the $B$-field confines the plasma laterally due to $J \times B$ effect (Joule heating). Hence the increase in electron temperature relative to the field-free case observed in Figure 7 could be caused by adiabatic compression of the plasma by the $B$-field (Harilal et al., 2004). However, the temperature presented in Figure 7 is time averaged over $2000 \mathrm{~ns}$ therefore the instantaneous temperature at earlier time is expected to be much higher.

We also estimated spatial evolution of density in a timeintegrated manner using Stark broadening of an isolated $\mathrm{Sn}$

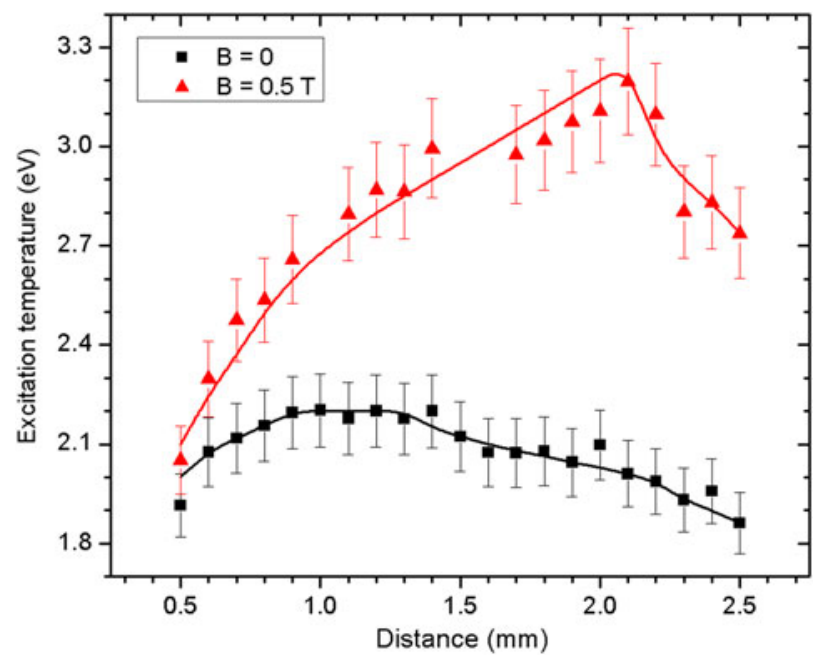

Fig. 7. The plasma excitation temperature with respect to distance from the target.

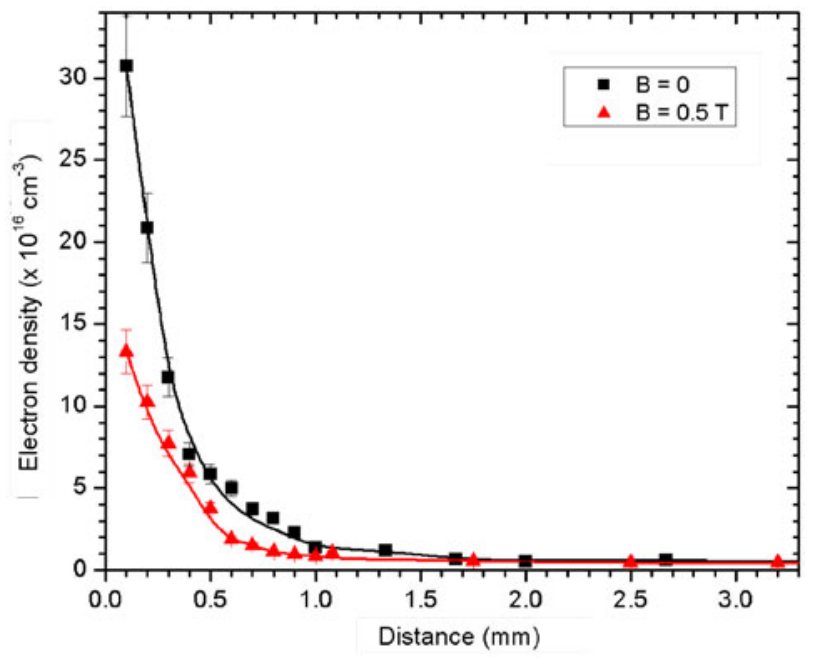

Fig. 8. The plasma density with respect to distance from the target.

line. Stark broadening of spectral lines in plasmas is caused by collisions of charged species, resulting in both a broadening of the line and a shift in the peak wavelength. The FWHM of Stark broadened lines is related to the electron number density $n_{\mathrm{e}}$ by

$$
\Delta \lambda_{1 / 2}=2 W_{\mathrm{p}}\left(\frac{n_{\mathrm{e}}}{10^{16}}\right)\left[1+1.75 G\left(\frac{n_{\mathrm{e}}}{10^{16}}\right)^{1 / 4}\left(1-\frac{3}{4} N_{\mathrm{D}}^{-1 / 3}\right)\right]
$$

where $N_{\mathrm{D}}$ is the number of particles in the Debye sphere. $W_{\mathrm{p}}$ is the electron impact parameter in nanometers, and $G$ is the ion impact parameter; $W_{\mathrm{p}}$ and $G$ are functions of temperature. The ion broadening parameters $G$ is typically negligible for nonhydrogenic ions compared with electron contribution and can be neglected. The spectroscopic parameters are obtained from NIST data base and Alonso-Medina and Colon (2008). The density has been estimated from the time integrated line intensities with the duration of $2000 \mathrm{~ns}$. We have selected the line broadened profile of the Sn II line at $645.4 \mathrm{~nm}$ for the density measurements.

Figure 8 shows the experimentally measured electron density for the same conditions as the case of plasma temperature measurements. One can see from Figure 8 that the plasma density reduces significantly with the application of $0.5 \mathrm{~T}$ axial $B$-field at shorter distances and the $B$-field has lesser influence at larger distances. In the field-free case, the electron density falls from $3.1 \times 10^{17} \mathrm{~cm}^{-3}$ at $0.1 \mathrm{~mm}$ to $1.5 \times$ $10^{16} \mathrm{~cm}^{-3}$ at $1 \mathrm{~mm}$, while the corresponding values are $1.4 \times 10^{17}$ at $0.1 \mathrm{~mm}$ and $1.4 \times 10^{16}$ in the presence of the axial $B$-field. The density reduction at shorter distances from the plasma could be due to increased recombination caused by magnetic field plasma confinement. However, we noticed significant intensity enhancement for Sn II in the presence of $B$ field, especially at shorter distances (Fig. 6) where excitation temperature differences (with and 
without $B$ ) is minimal (Fig. 7). It has to be mentioned that all the measurements given in the figures are time averaged. But plasma $\beta$ is highly dynamic and hence time-resolved studies are needed to explain these, which is a scope of future study.

We have considered the plasma to be in local thermal equilibrium (LTE) for the estimation of temperature from the spectral data. We verified this assumption using the McWhirter condition (Bekefi, 1976). In the present experiment, the plume is believed to be in LTE because the observed values of $n_{\mathrm{e}}$ are always greater than $3.15 \times 10^{15} \mathrm{~cm}^{-3}$.

The confinement of the plasma is apparent in the presence of axial $B$-field (Fig. 1). The plasma expansion stops when the plasma pressure becomes equal to magnetic pressure or $\beta=1$. The magnetic pressure can be estimated as $p_{B}=\frac{1}{2}$ $B_{\mathrm{o}}{ }^{2} / \mu_{\mathrm{o}}$. For $0.5 \mathrm{~T} B$-field the estimated magnetic pressure is $9.9 \times 10^{4} \mathrm{~Pa}$. The plasma pressure is $p_{\mathrm{eo}}=n_{0} k T_{\mathrm{e}}$. The estimated time integrated plasma pressure at 0.5 and $2.5 \mathrm{~mm}$ from the surface is $1.31 \times 10^{4}$ and $0.45 \times 10^{4} \mathrm{~Pa}$, respectively. However, the plasma pressure is significantly higher at early times and shorter distances. Therefore at larger distances the reduction in temperature and density will lead to reduced plasma pressure and hence confinement of the plasma takes place.

\section{CONCLUSIONS}

The interaction between an expanding laser-produced plasma and a $0.5 \mathrm{~T}$ axial $B$-field has been studied using gated imaging, optical emission spectroscopy and PIC modeling. ICCD images showed focusing of the plasma plumes in the presence of axial $B$-field. It was shown that the ICCD images can be reproduced by the PIC simulation images. The spectroscopic studies in the presence of $B$-field showed enhancements in background continuum as well as an increase in intensity from ionic lines while neutral lines are less affected. The spatial extension of certain lines was considerably increased while some of them were suppressed in the presence of the $0.5 \mathrm{~T}$ axial $B$-field.

Space resolved measurements of electron density and temperature were carried out using emission lines of singly ionized tin. It was found that the plasma temperature increases with distance in the presence of an axial magnetic field. However, the plasma density is found to be deceased at shorter distances from the target in the presence of $B$-field. The increase in temperature in the presence of the axial magnetic field could be related to confinement heating of $B$-field, while the reduction in density is explained due to increased recombination caused by magnetic field plasma confinement.

\section{ACKNOWLEDGMENTS}

This work was supported in part by the U.S. National Science Foundation (PIRE project) and co-financed by the European Regional Development Fund, the European Social Fund and the state budget of the Czech Republic (Project HiLASE: CZ.1.05/2.1.00/
01.0027, Project DPSSLasers: CZ.1.07/2.3.00/20.0143, Project Postdok: CZ.1.07/2.3.00/30.0057). Pacific Northwest National Laboratory, a multi-program national laboratory operated by Battelle for the U.S. Department of Energy under Contract DE-AC05-76RL01830

\section{REFERENCES}

Alonso-Medina, A. Colon, C. (2008). Measured stark widths of several Sn I and Sn II spectral lines in a laser-induced plasma. Astrophys. J. 672, 1286-1291.

BAKSHI, V. (2009). EUV Lithography. Bellingham, WA/Hoboken, NJ: SPIE and John Wiley \& Sons, Inc.

BALK, M. (2007) 3rd European User Group Meeting Technical Sessions, 13-14 September 2007 on Lake Tegernsee, Germany. “CST PARTICLE STUDIO” (CST AG). https://www.cst. com/Content/Events/UGM2007/04-Balk.pdf

Bekefi, G. (1976). Principles of Laser Plasmas. New York: Wiley-Interscience.

Coons, R.W., Harilal, S.S., Campos, D. \& Hassanein, A. (2010). Analysis of atomic and ion debris features of laser-produced Sn and Li plasmas. J. Appl. Phys. 108, 063306.

Dimonte, G. \& Wiley, L.G. (1991). Dynamics of exploding plasmas in a magnetic field. Phys. Rev. Lett. 67, 1755-1758.

Gekelman, W., Collette, A. \& Vincena, S. (2007). Threedimensional current systems generated by plasmas colliding in a background magnetoplasma. Phys. Plasmas 14, 062109.

Griem, H.R. (1997). Principles of Plasma Spectroscopy. Cambridege, UK: Cambridege University Press.

Harilal, S.S., O'shay, B. \& Tillack, M.S. (2005a). Spectroscopic characterization of laser-induced tin plasma. J. Appl. Phys. 98, 013306.

Harilal, S.S., O’Shay, B., Tillack, M.S., Bindhu, C.V. \& NaJMaBADI, F. (2005b). Fast photography of a laser generated plasma expanding across a transverse magnetic field. IEEE Trans. Plasma Sci. 33, 474-475.

Harilal, S.S., Tillack, M.S., O’Shay, B., Bindhu, C.V. \& NaJMaBADI, F. (2004). Confinement and dynamics of laser-produced plasma expanding across a transverse magnetic field. Phys. Rev. E 69, 026413.

Harilal, S.S., Tillack, M.S., Tao, Y., O’Shay, B., Paguio, R. \& NiKroo, A. (2006). Extreme-ultraviolet spectral purity and magnetic ion debris mitigation by use of low-density tin targets. Opt. Lett. 31, 1549-1551.

Joshi, H.C., Kumar, A., Singh, R.K. \& Prahlad, V. (2010). Effect of a transverse magnetic field on the plume emission in laserproduced plasma: An atomic analysis. Spectrochimica Acta B 65, 415-419.

Kumar, A., George, S., Singh, R.K., Joshi, H. \& Nampoori, V.P.N. (2011a). Image analysis of expanding laser-produced lithium plasma plume in variable transverse magnetic field. Laser Part. Beams 29, 241-247.

Kumar, A., Singh, R.K. \& Joshi, H. (2011b). Effect of transverse magnetic field on the laser-blow-off plasma plume emission in the presence of ambient gas. Spectrochim. Acta B 66, 444-450.

Kumar, R., Singh, R.K. \& Kumar, A. (2012). Effects of magnetic field on oscillatory structures in laser-blow-off plasma. Phys. Lett. A 377, 93-98. 
National Institute of Standards and Technology (NIST). www.nist. gov

Neogi, A. \& Thareja, R.K. (1999). Laser-produced carbon plasma expanding in vacuum, low pressure ambient gas and nonuniform magnetic field. Phys. Plasmas 6, 365-371.

Pagano, C. \& Lunney, J.G. (2010). Lateral confinement of laser ablation plasma in magnetic field. J. Phys. D: Appl. Phys. 43, 305202.

Pandey, P.K. \& Thareja, R.K. (2011). Plume dynamics and cluster formation in laser-ablated copper plasma in a magnetic field. J. Appl. Phys. 109, 074901

RAI, V.N., RAI, A.K., YUEH, F.Y. \& Singh, J.P. (2003). Optical emission from laser-induced breakdown plasma of solid and liquid samples in the presence of a magnetic field. Appl. Opt. 42, 2085.

Roy, A., Harilal, S.S., Polek, M.P., Hassan, S.M., Endo, A. \& HASSANEIN, A. (2014a). Influence of laser pulse duration on extreme ultraviolet and ion emission features from tin plasmas. Phys. Plasmas 21, 033109.

Roy, A., Hassan, S.M., Harilal, S.S., Endo, A., Mocek, T. \& HasSANEIN, A. (2014b). Extreme ultraviolet emission and confinement of tin plasmas in the presence of a magnetic field. Phys. Plasmas 21, 053106.

Schwarz, H. \& Hora, H. (1971). In Laser Interaction and Related Plasma Phenomena, (Schwarz, H. and Hora, H., Eds.), p. 301. New York: Plenum Press.

Shen, X.K., Lu, Y.F., Gebre, T., Ling, H. \& Han, Y.X. (2006). Optical emission in magnetically confined laser-induced breakdown spectroscopy. J. Appl. Phys. 100, 053303.
Sizyuk, V., Hassanein, A. \& BaKshi, V. (2007). Modeling and optimization of debris mitigation systems for laser and dischargeproduced plasma in extreme ultraviolet lithography devices. J. Micro/Nanolithogr. MEMS, MOEMS 6, 043003.

Tsui, Y.Y., Minami, H., Vick, D. \& Fedosejevs, R. (2002). Debris reduction for copper and diamond-like carbon thin films produced by magnetically guided pulsed laser deposition. J. Vac. Sci. Technol. A 20, 744-747.

Ueno, Y., Soumagne, G., Sumitani, A., Endo, A., Higashiguchi, T. \& Yugami, N. (2008). Reduction of debris of a CO2 laserproduced Sn plasma extreme ultraviolet source using a magnetic field. Appl. Phys. Lett. 92, 211503.

Vanzeeland, M., Gekelman, W., Vincena, S. \& Dimonte, G (2001). Production of Alfvén waves by a rapidly expanding dense plasma. Phys. Rev. Lett. 87, 105001.

Vanzeeland, M., Gekelman, W., Vincena, S. \& Maggs, J. (2003). Currents and shear Alfvén wave radiation generated by an exploding laser-produced plasma: Perpendicular incidence. Phys. Plasmas 10, 1243-1252.

Wu, T., Wang, Lu, X.H. \& Lu, P. (2012). Debris mitigation power of various buffer gases for $\mathrm{CO} 2$ laser produced tin plasmas. J. Phys. D: Appl. Phys. 45, 475203.

Ye, C., Cheng, G.J., TAO, S. \& Wu, B.X. (2013). Magnetic field effects on laser drilling. J. Manuf. Sci. Eng. 135, 061020.

Yuspeh, S., Tao, Y., Burdt, R.A., Tillack, M.S., Ueno, Y. \& NaJMABADI, F. (2011). Dynamics of laser-produced Sn microplasma for a high-brightness extreme ultraviolet light source. Appl. Phys. Lett. 98, 201501. 